\title{
Hitos y transformaciones en el área de Higiene y Epidemiología
}

\section{Landmarks and changes in the sphere of Hygiene and Epidemiology}

\section{Vivian Noriega Bravo}

Máster en Salud Pública. Profesora Auxiliar. Escuela Nacional de Salud Pública. La Habana, Cuba.

\section{RESUMEN}

Desde las primeras etapas en la formación del sistema revolucionario de salud en Cuba, se ha asistido a un proceso continuo de perfeccionamiento en el que las transformaciones acontecidas, particularmente en políticas y estrategias, responden a las demandas del contexto en cada momento histórico. La importancia conferida a la prevención y control de enfermedades y daños a la salud ha hecho de los servicios higiénico-epidemiológicos una prioridad. Resultado de la revisión bibliográfica y la experiencia de la autora, el presente trabajo muestra una breve reseña de la conformación del área de Higiene y Epidemiología, en especial de los centros y unidades que la representan a nivel provincial y municipal;

conjuntamente con algunas consideraciones sobre hechos y momentos que han tenido significación en los cambios acontecidos en su configuración organizativa.

Palabras clave: Área de Higiene y Epidemiología, centros y unidades de Higiene y Epidemiología, servicios higiénico-epidemiológicos, estructura organizativa, cambios organizativos, Cuba.

\section{ABSTRACT}


Since the first stages of inception of the revolutionary health system in Cuba, there has been a continuous process of improvement in which changes, particularly in policies and strategies, responded to the demands of each specific historical period. The importance attached to prevention and control of diseases and health deterioration has turned the hygienic-epidemiological services into a priority. As a result of the literature review made by and the own experience of the author, the present paper showed a brief account of the conformation of the Hygiene and Epidemiology field, specially the centers and units representing this sector at municipal and provincial levels, together with some considerations on events and periods that have been significant for the changes made in the organization of this branch.

Key words: Hygiene and Epidemiology area, hygiene and epidemiology centers and units, hygienic-epidemiological services, organizational structure, organizational changes, Cuba.

\section{NTRODUCCI ÓN}

Las malas condiciones de vida y el bajo nivel de bienestar tanto material como cultural de la población cubana antes del triunfo de la Revolución en 1959, se veían reflejados en críticos problemas de salud como eran las elevadas tasas por enfermedades infectocontagiosas, elevada mortalidad del niño menor de un año a causa de enfermedades prevenibles, unido a una legislación sanitaria anacrónica e insuficiente, carencia de una estructura organizativa de la salud pública y ausencia de voluntad política para transformar la situación.

Las actividades de protección y atención a los problemas higiénicos y epidemiológicos eran prácticamente inexistentes, a pesar de contar con ciertos servicios estatales que debían cumplir esa misión, vinculados al llamado Ministerio de Salubridad y Asistencia Social. ${ }^{1}$

Luego del triunfo revolucionario, el Estado comenzó grandes y esforzadas transformaciones en el orden económico, político y social y de igual modo, desde la formación del Sistema Nacional de Salud (SNS) se reconocen cambios que ha devenido en un proceso continuo de perfeccionamiento en concordancia con el nivel de desarrollo alcanzado por el país y que ha contribuido al mejoramiento sostenido de la salud de los cubanos.

Con la designación del Ministerio de Salud Pública, órgano de gobierno encargado del estudio de los problemas de la salud del pueblo y de planificar y ejecutar las acciones de fomento, protección y recuperación de la salud, orientar y crear condiciones óptimas que aseguren generaciones sanas, tanto en lo físico como en lo mental; se creó una subsecretaría, en la práctica un viceministerio, ocupada de las actividades de protección del hombre frente a las agresiones del ambiente, así como de los medios técnicos y científicos para actuar consecuentemente en su control, ${ }^{1}$ y que no son más que los pilares básicos del trabajo para la Higiene y Epidemiología. 
Aunque la producción de servicios higiénico-epidemiológicos en Cuba se enmarque con la creación de los Centros de Higiene y Epidemiología, es justo referir el papel desempeñado por las llamadas Unidades Sanitarias existentes en el país hasta 1960. Estas "estructuras organizativas" fueron creadas a partir de las J efaturas Locales de Sanidad y estaban definidas para realizar el control epidemiológico y de la higiene del ambiente físico, específicamente en la lucha contra enfermedades transmisibles como la tuberculosis, la fiebre tifoidea, el tétanos, la rabia y la enfermedad diarreica aguda, entre otras. A decir de la Dra. Martínez Calvo, refiriéndose a la Unidad Sanitaria de Marianao, las acciones allí desplegadas se convirtieron en precursoras de las que se desarrollaron luego, en las estrategias sanitarias de las dos primeras décadas del SNS. ${ }^{2}$

Los cambios experimentados en la estructura organizativa de las instituciones del sector de la salud y los servicios brindados por estas, han respondido a las demandas del contexto en cada momento histórico y los ocurridos en Higiene y Epidemiología no son una excepción. Sus instituciones son el reflejo de una diferenciación interna del sistema, en el cual la prioridad alcanzada por la prevención y control de enfermedades y daños a la salud, ha determinado en gran medida su configuración a un nivel de especialización capaz de satisfacer las necesidades de la población en este orden y en correspondencia con las exigencias de la Salud Pública Cubana.

Es propósito del presente trabajo reseñar algunos de los momentos y hechos que han tenido significación en el quehacer del Área de Higiene y Epidemiología, algunos de los cuales han devenido en cambios en la estructura y organización de sus principales instituciones a nivel provincial y municipal.

\section{NTEGRACI ÓN DE LA RED CURATI VO-PREVENTI VA}

Entre las principales estrategias en materia de salud desarrolladas en la década del 70, estuvieron aquellas dirigidas a incrementar el nivel inmunitario de la población menor de 15 años y a la lucha contra las enfermedades infecto-contagiosas, con la participación popular.

Si bien pueden ser numerosos los ejemplos, sólo se mencionaran dos estructuras (departamentos ejecutivos autónomos y asesorados por organismos internacionales) que ejecutaron algunas de esas estrategias: el Servicio Nacional de Erradicación del Paludismo y la Campaña Anti-Aedes aegypti. La labor desplegada por la primera, permitió interrumpir la transmisión de la malaria en el país y la otra, tuvo a su cargo el desarrollo de un plan de acción dirigido a erradicar el mosquito transmisor de la fiebre amarilla, primero territorialmente, luego con acciones integradas a los servicios generales de salud y que con otra concepción, aún cumple una importante misión dentro del sistema.

El fortalecimiento del aparato organizativo para la creación de un sistema de salud único e integrado por una red de instituciones preventivo-curativas debidamente diferenciadas en su gestión, enmarcó la creación en 1962 de los Centros de Higiene y Epidemiología (uno por provincia excepto la antigua provincia de Oriente que tenía dos) a los que se le definieron funciones específicas, en lo referente al control de las enfermedades transmisibles, saneamiento del medio ambiente, la higiene de trabajo, del escolar, la nutrición y la educación sanitaria. ${ }^{1}$

El I Forum Nacional de Higiene y Epidemiología celebrado en septiembre de ese mismo año, se reconoce como el primer y más importante paso para desarrollar, 
ordenar y normar las actividades de promoción de salud y prevención de enfermedades. En este Forum, no sólo se analizaron los primeros logros y se establecieron los procedimientos para el control sanitario y los métodos de lucha contra las enfermedades transmisibles, sino que los materiales de tipo técnico y metodológico aprobados en sus sesiones, fueron editados y convertidos en normas generales de la especialidad y guías para el quehacer diario del reducido número de especialistas con que se contaba.

Con la puesta en funcionamiento del policlínico integral se introdujeron los servicios de atención epidemiológica y al medio ambiente, como parte de una atención integral al hombre y su entorno, a partir de la idea del policlínico como unidad básica para la atención ambulatoria a la población, bajo una orientación profiláctica de las acciones y organizada para enfrentar, mediante programas y campañas, las principales enfermedades transmisibles.

En esa etapa los Centros de Higiene y Epidemiología, llamados Laboratorios Provinciales, tuvieron un importante papel en la lucha contra las enfermedades diarreicas agudas, en la realización del diagnóstico microbiológico de sus agentes causales, pesquisa de portadores y la implementación de técnicas para el control microbiológico y químico del agua, la leche y otros alimentos; en el desarrollo de los programas de lucha contra la rabia, la tuberculosis pulmonar y el paludismo.

Cabe reconocer además, lo que significó la figura del trabajador sanitario, hoy técnico de higiene y epidemiología (THE), quien surgió como parte de la política de la revolución de llevar las acciones preventivas hasta los lugares más intrincados del país. Este trabajador desarrolló sus actividades básicamente en la solución de los problemas higiénicos y aunque no siempre podía interrelacionarlos con las consecuencias epidemiológicas que tenían sobre la salud del hombre, su labor fue por mucho tiempo, un elemento de integración con el resto de las unidades del sistema para llevar a cabo campañas periódicas de vacunación, de higienización, movilización de la población a la realización de exámenes masivos para la detección precoz de enfermedades, en la difusión de mensajes educativos, vigilancia de personas en riesgo y otras importantes tareas.

\section{NSTI TUCI ONALI ZACI ÓN Y CONSOLI DACI ÓN DEL SI STEMA DE SALUD}

En los años 70, una vez atendidos los problemas de salud más urgentes, el sistema de salud entró en una fase superior de consolidación de sus instituciones, de perfeccionamiento de las normativas y el desarrollo de los programas básicos ejecutados en las áreas de salud. Se implementó un modelo de atención cualitativamente superior, el de Medicina en la Comunidad, con el que se ampliaron los logros que ya se tenían en el control y erradicación de enfermedades trasmisibles y se comenzaron a obtener los primeros resultados en la prevención y control de enfermedades no transmisibles.

En el frente de Higiene y Epidemiología se valoraron los aspectos críticos y se trazaron proyecciones de trabajo, entre ellas, la introducción de cambios estructurales dentro del viceministerio y los niveles inferiores, que permitieran acometer mejor las tareas.

El II Forum de Higiene y Epidemiología celebrado en septiembre de 1974, se señala como un hecho importante y pese a que las proyecciones de trabajo exigían 
reforzar la estructura y funciones del viceministerio correspondiente a un nivel altamente especializado, por el contrario, se decidió su supresión (1977), quedando la Dirección Nacional de Higiene subordinada directamente al ministro y la de Epidemiología transformada en un departamento dependiente del Viceministerio de Asistencia Médica.

En tanto, los niveles jerárquicos y de servicios provincial y municipal se fortalecieron con la nueva división político-administrativa del país, la que dio paso a las Direcciones Provinciales de Salud y con ello a los actuales Centros Provinciales de Higiene y Epidemiología ( $\mathrm{CPHE}$ ) dependiente de estas y que tienen su representación en los centros y unidades municipales de igual denominación (CUMHE), según cuenten o no con laboratorio sanitario en su estructura. Todos estos centros y unidades son instituciones que hoy representan al Área de Higiene y Epidemiología (figura).

\section{LA SITUACI ÓN EPI DEMI OLÓGI CA DE LA ENFERMEDAD MENI NGOCÓCCI CA Y EL DENGUE HEMORRÁGI CO}

En la década de 1981-1990 ocurrieron importantes eventos que indujeron a cambios en el Área de Higiene y Epidemiología, sobre todo en el nivel provincial e inferior y a partir de lo cual, se logra la consolidación en la organización de sus servicios.

La enfermedad meningocóccica de tipo B constituyó en esos años el principal problema epidemiológico para Cuba. El aumento progresivo del número de casos a partir de 1978 y la pérdida anual de decenas de vidas en la población infantil, requirió un intenso programa de lucha contra la enfermedad durante un tiempo prolongado. Los C-UMHE enfrentaron la epidemia, sus consecuencias y acometieron múltiples actividades de control epidemiológico: estudio de enfermos, de contactos convivientes, en instituciones cerradas, pesquisa de portadores; todo ello con la finalidad de obtener un diagnóstico correcto, proteger a personas susceptibles y educar sanitariamente a la población. ${ }^{3}$

En todos los CPHE se conformaron equipos encargados de asesorar, controlar y ejecutar todas las actividades de control de foco, tras la notificación de un caso sospechoso o confirmado de la enfermedad y funcionaron equipos de Vigilancia Personal Antimeningocóccica que operaban en centros fijos y unidades móviles durante las $24 \mathrm{~h}$ del día. Al CPHE de Ciudad de La Habana se le reconoce además, como la institución donde se elaboraron los planes de emergencia para enfrentar esta problemática y por haber contribuido al fortalecimiento de los laboratorios de Microbiología del país.

Otro evento fue la epidemia de dengue hemorrágico de 1981, distinguida como el acontecimiento de mayor connotación en la historia del dengue en Cuba. Durante la epidemia, fue necesario realizar una intensa campaña para reducir a niveles muy bajos los índices de infestación vectorial y eliminar la transmisión de la enfermedad para no lamentar un número mayor de muertes.

La situación vivida, tuvo como respuesta no sólo la implementación de un programa nacional dirigido a la erradicación del mosquito Aedes aegypti, sino a que se tomaron decisiones administrativas y organizativas a nivel del MINSAP para la creación de una infraestructura, técnica y material, que potenciara el trabajo en el Área de Higiene y Epidemiología, particularmente en el "control de vectores." Es así 
que en enero de ese mismo año, se asumió de nuevo un viceministerio independiente de Asistencia Médica y se fortaleció la red de instituciones del Área.

La experiencia adquirida en el enfrentamiento a estas y otras epidemias, la búsqueda de una atención más calificada al hombre en su ambiente y de una mayor efectividad en la gestión de los C-UMHE, fue la premisa para que en 1984, se redefiniera la estructura, organización y funciones de dichas instituciones. ${ }^{4}$

Fue así, que el personal responsabilizado con la inspección sanitaria estatal (ISE) fue reubicado desde las áreas de salud hacia los centros y unidades municipales; de esta manera, las actividades de inspección y lucha antiepidémica sólo eran planificadas, ejecutadas y controladas por estas instituciones; mientras que las acciones de prevención, higiénico-epidemiológicas y de educación para la salud, eran planificadas, asesoradas y controladas por el aparato de Higiene y Epidemiología a todos los niveles del sistema. Con la adopción de esta nueva forma de trabajo se pretendía obtener:

- Más efectividad de la lucha antiepidémica y la ISE.

- Viabilidad en las coordinaciones y el control higiénico-epidemiológico hacia otros organismos del Estado.

- Vinculación de los programas de prevención de salud con la comunidad, a través de sus organizaciones.

- Uniformidad de criterios organizativos, metodológicos y de supervisión.

- Adecuada planificación de los recursos humanos y materiales necesarios y disponibles para la prestación de servicios, docencia e investigación.

- Apoyo a la gestión de la red del servicio higiénico-epidemiológico del país, mediante las direcciones nacionales e instituciones de investigación del Área.

- Funcionabilidad y operatividad de las coordinaciones internas con otras áreas del MINSAP para facilitar el trabajo en equipo.

- Asimilación de los avances científicos y técnicos generados por el desarrollo económico y social del país, sin necesidad de cambios estructurales de peso en la organización del trabajo de Higiene y Epidemiología.

Se plantea que otro elemento tenido en cuenta, fue el inicio de la incorporación del médico de familia, con el que se ampliaba las posibilidades del accionar higiénicoepidemiológico en el área de salud, dada sus potencialidades para modificar estilos de vida, orientar hábitos saludables, controlar riesgos y prevenir enfermedades y estar también responsabilizado con la ejecución de acciones de vigilancia de la higiene en su territorio.

Si bien esta forma organizativa no estuvo exenta de críticas, por considerarla predominantemente verticalizada y que quebrantó la labor integral realizada hasta entonces por el policlínico, se le reconoce su contribución al mejoramiento del trabajo de los CPHE y los C-UMHE y por consiguiente, a la obtención de resultados exitosos en los programas de control epidemiológico. 


\section{MPLEMENTACI ÓN DE UNA NUEVA ESTRATEGI A SANITARI A Y LA SITUACI ÓN DE LA NEUROPATÍ A EPI DÉMI CA}

En el marco de la profunda crisis económica debido a la caída del campo socialista y el recrudecimiento del bloqueo económico por parte del gobierno de Estados Unidos en el decenio de los 90, el sector de la salud tuvo que encarar los cambios comprobados en el perfil de morbilidad y mortalidad, el deterioro ambiental y sus implicaciones en la salud de la población y la emergencia de nuevos problemas de salud. De ahí que la concepción, implementación y ejecución exitosa de una estrategia sanitaria orientada epidemiológicamente, los Ilamados Objetivos, Propósitos y Directrices para incrementar la salud de la población cubana 19922000 (OPD-2000), a fin de mantener y mejorar los indicadores de salud de la población, constituyó para el MINSAP su mayor reto y más importante tarea. Se tenía como antecedente, entre otros elementos, la amplia cobertura de médico y enfermera de la familia y las múltiples actividades encaminadas a la lucha contra las enfermedades no transmisibles y los accidentes, que aisladamente se venían realizando. ${ }^{5}$

En correspondencia, se llevaron a cabo importantes transformaciones, aunque no uniformes, en la organización del trabajo de los centros y unidades de Higiene y Epidemiología del país. Un ejemplo de los cambios estructurales realizados en el CPHE de La Habana, lo fue la creación de la Vicedirección de Enfermedades no Trasmisibles (hoy Vicedirección Primera) la que se conformó con epidemiólogos que en su mayoría ya venían trabajando en el Departamento de Control Epidemiológico, en un grupo técnico para el estudio de las principales causas de mortalidad en la población e integrando comisiones de trabajo multidisciplinarias; y además, con higienistas de los diferentes perfiles procedentes de la Vicedirección de Higiene (denominada luego Vicedirección de Salud Ambiental). Esta última quedó integrada por especialistas y THE que accionarían integralmente en actividades de higiene comunal, de los alimentos y nutrición, ocupacional y escolar.

Cabe decir, que se confrontaron dificultades para la puesta en funcionamiento de los dos grupos de trabajo que componían la vicedirección: el de Vigilancia Epidemiológica y el de Evaluación, pues aunque en teoría se reubicaron los especialistas buscando integralidad en el desempeño y avances en el orden científico-técnico; en la práctica, se hizo una distribución acrítica del personal que limitó la efectividad del cambio.

Uno de los problemas más difíciles enfrentados por el sector de la salud a inicios de 1992, fue el incremento progresivo en el número de casos de lo que después se conoció como la epidemia de neuropatía.

Su enfrentamiento, como en todas, dejó enseñanzas, en este caso la necesidad de redefinir elementos estructurales y estrategias dirigidas a hacer más efectiva y eficiente la respuesta del Área de Higiene y Epidemiología ante la situación sanitaria que vivía el país.

A decir del compañero Fidel Castro, en las palabras de clausura del Taller Internacional sobre Neuropatía Epidémica:

..se descubrió que en Epidemiología teníamos lagunas y atrasos en algunos laboratorios diagnósticos, inexperiencia, que no contábamos con toda la tecnología, con todos los equipos necesarios. Existían debilidades en el campo de las investigaciones. ${ }^{6}$ 
Con la finalidad de revertir esa situación y responder a las indicaciones del máximo líder, se implementó en el nivel central (1993) la Unidad de Análisis y Tendencia en Salud (UATS), de la que se estableció luego una red con unidades municipales y provinciales. El interés, ampliar el enfoque hacia un sistema de vigilancia en salud con mayor integración de la información, mayor nivel de análisis y mejor utilización de la información generada para la toma de decisiones, oportuna y con base científica. $^{7}$

La creación de estas unidades puede decirse que enmarcó una etapa de progreso para el viceministerio de Higiene y Epidemiología, así como para sus centros y unidades representativas, ya que propició un amplio proceso de desarrollo tecnológico, de superación científico-técnica y promovió un estilo de trabajo con una visión multidisciplinaria más profunda.

\section{DESCENTRALI ZACI ÓN Y FORTALECI MI ENTO DEL NI VEL LOCAL}

Atendiendo a la situación económica, política y social existente en el país en 1993, se planteó un cambio importante en los objetivos y estrategias de trabajo del MINSAP, en la que se privilegió entre otros, la promoción de la salud y la prevención de enfermedades, el fortalecimiento del nivel local acorde al desarrollo de las estructuras de gobierno denominadas Consejos Populares, el aprovechamiento del potencial de recursos humanos calificados para transformar los estilos y las condiciones de vida de los diferentes grupos poblacionales y la adopción de nuevos estilos de dirección, más ágiles y operativos en todos los niveles del SNS.

En correspondencia con este proceso, se crearon al año siguiente, las subdirecciones de Higiene y Epidemiología en los policlínicos, con lo que se transformó la estructura organizativa mantenida por casi una década, y se asumió nuevamente una dimensión del trabajo higiénico-epidemiológico que posibilita un mayor acercamiento de las decisiones al lugar donde se producen los problemas de salud.

En la nueva estructura las actividades se organizan en los municipios en dos niveles: el primero se corresponde con los médicos de familia, los Grupos Básicos de Trabajo y el Consejo Popular; el segundo, con el C-UMHE que abarca integralmente la totalidad del territorio y además, actúa como nivel de referencia para las áreas de salud, las que jerarquiza técnica y metodológicamente. ${ }^{8}$

Estas subdirecciones se conformaron en su mayoría con personal procedentes de los propios centros y unidades de Higiene y Epidemiología, muchos de ellos bien calificados, otros lamentablemente menos aptos para asumir el reto de fomentar el enfoque clínico, epidemiológico y social como guía fundamental para la toma de decisiones a ese nivel. Paulatinamente, las plazas de subdirectores fueron ocupadas casi en su totalidad por especialistas en Medicina General Integral (MGI).

Sin embargo, los "reajustes" realizados en los últimos años para mantener la vitalidad de la atención en el nivel primario, ha afectado seriamente la permanencia y selección de los $\mathrm{MGI}$ en esa actividad, y actualmente, constituye un problema y una prioridad, la carencia de especialistas calificados y con experiencia para actuar a ese nivel y enfrentar la situación higiénico-sanitaria existente en el país. 
Se concluye con la consideración de que los cambios implementados, no siempre acertados, en la estructura organizativa y la dinámica de trabajo en el Área de Higiene y Epidemiología desde su creación, han respondido a las exigencias en el cumplimiento de sus funciones y el compromiso de acometer de manera exitosa nuevos y mayores retos.

\section{REFERENCI AS BI BLI OGRÁFICAS}

1. del Puerto Quintana C, Ferrer García H, Toledo Curbelo G. Higiene y Epidemiología. Apuntes para la Historia. La Habana: Editora Palacio de las Convenciones; 2002.

2. Martínez Calvo S. El Dr. Pedro Nogueira Rivero: un salubrista cubano. Rev Cubana Salud Pública [serie en Internet]. 2004 [citado 22 Ago 2008]; 30(2).

Disponible en: http://www.bvs.sld.cu/revistas/spu/indice.html

3. Valcárcel Novo M, Rodríguez Cruz R, Terry Molinert H. La Enfermedad Meningocócica en Cuba. Cronología de una epidemia. La Habana: Editorial Ciencias Médicas; 1991.

4. Ministerio de Salud Pública de Cuba. Organización de las instituciones higiénicoepidemiológicas (CPHE, CMHE y UMHE) del Sistema Nacional de Salud. Documento 1. La Habana: MINSAP; 1984.

5. Ministerio de Salud Pública de Cuba. Objetivos, Propósitos y Directrices para incrementar la salud de la población cubana 1992-2000. La Habana: MINSAP; 1992.

6. Castro Ruz F. Discurso pronunciado en la clausura del Taller Internacional sobre Neuropatía Epidémica. La Habana, 15 de julio de 1994. [citado 22 Ago 2008].

Disponible en: http://www.cuba.cu/gobierno/discursos/1994/esp/f150794e.html

7. Rodríguez Milord D. Práctica de la vigilancia en Cuba. La Habana: Editorial Ciencias Médicas; 1997.

8. Ministerio de Salud Pública de Cuba. La integración de la Higiene y la Epidemiología a la atención primaria de salud. La Habana: MINSAP; 1995.

Recibido: 22 de septiembre de 2008.

Aprobado: 8 de octubre de 2008.

Vivian Noriega Bravo. Escuela Nacional de Salud Pública. Calle Línea esq. I. El Vedado10400. La Habana, Cuba.

E-mail: vivy@infomed.sld.cu 
